\title{
Search for GRB counterparts using the ARGO-YBJ experiment in shower mode
}

\author{
S.Z Chen ${ }^{1}$, H.H He, Z Cao, F.R Zhu, X.X Li \\ on behalf of ARGO-YBJ collaboration \\ Key Lab. of Particle Astrophysics, Institute of High Energy Physics, CAS, Beijing 100049, China
}

\begin{abstract}
In this paper, the effective area of the ARGO-YBJ experiment to detect the gamma rays with energy range from 10 $\mathrm{GeV}$ to $1 \mathrm{TeV}$ for different incident zenith angles $(0,10,20,30,40$ degrees $)$ is given respectively. The optimal event selections to search for GRB counterparts with different cutoff energy models (Ecut $=100 \mathrm{GeV}$ and $1 \mathrm{TeV}$ ) are studied. Using these selections and their corresponding angular resolutions the searches are done within two hours around the GRB trigger time via different time windows. Finally, the result for the $20 \mathrm{GRBs}$ in the field of view of ARGO between July 2006 and July 2008 is presented, and the fluence upper limit for each GRB is set at $99 \%$ confidence level.
\end{abstract}

Keywords: GRB, gamma-ray, EAS, cosmic ray

PACS: $98.70 . \mathrm{Rz}$, 95.85.Pw, 96.40.Pq, 95.85.Ry

\section{INTRODUCTION}

Gamma-ray bursts (GRBs) are short-lived bursts of gamma-ray photons. They are known to be isotropic with a nonthermal origin and divided into two classes(long and short). EGRET detected seven GRBs with photon energy ranging from $100 \mathrm{MeV}$ to $18 \mathrm{GeV}$ during both the prompt and afterglow phases[1,2], and no high energy cutoff was measured. Very high energy (VHE) emission up to $\mathrm{TeV}$ is predicted by several models during prompt and afterglow phases [3]. This can occur as a result of electron self-IC emission from the internal shock or the external forward/reverse shock. No conclusive detection was reported by previous searches for VHE emission from GRBs, with some positive evidences by Tibet $A S \gamma$ [4], Milagrito [5], HEGRA AIROBICC [6] and GRAND [7]. The Cerenkov detector MAGIC gave a very low upper limit between $85-1000 \mathrm{GeV}[8]$.

The ARGO-YBJ experiment [9], a collaboration among Italian and Chinese institutes, is located in Tibet, China. Its high altitude ( $4300 \mathrm{~m}$ a.s.l.) and full coverage of Resistive Plate Chambers (RPCs) over $5600 \mathrm{~m}^{2}$ enable the study of the high energy end of GRBs with an energy threshold as low as few hundreds of GeV. With the large field of view $(\sim 2 s r)$ and high duty cycle ( $>90 \%)$, the ARGO-YBJ experiment is one of the best satellite follow-up apparatuses.

\section{SIMULATION AND DATA ANALYSIS}

The effective area of the ARO-YBJ experiment for detecting gamma rays is studied by a full Monte Carlo simulation with CORSIKA6.502 and ARGOG-V144 (ARGO-YBJ simulation code) [10]. Five typical zenith angles $\left(\theta=0^{\circ}, 10^{\circ}\right.$, $20^{\circ}, 30^{\circ}, 40^{\circ}$ ) are considered in the simulation. The average effective area over different energy ranges (spectrum index -2.0) as a function of zenith angle $\theta$ could be well fitted with a function $A_{0} \cos ^{n} \theta$, with $n=14.26,10.56$ respectively, and consequently the effective area at any zenith angle could be obtained. At $\theta=20^{\circ}$ and starting from $\mathrm{E}=10 \mathrm{GeV}$, the effective area of the ARGO-YBJ experiment is about $3 m^{2}$ if the GRB cutoff energy Ecut is $100 \mathrm{GeV}$, while it reaches $100 \mathrm{~m}^{2}$ when Ecut $=1000 \mathrm{GeV}$ (see Fig. 1 ).

In the ARGO-YBJ experiment, the event hit multiplicity $N_{\text {hit }}$ corresponds to the primary energy. If a GRB Ecut is assumed, limiting $N_{\text {hit }}$ in the data analysis can greatly reject cosmic ray background which cannot be discriminated from gamma at low energies in the ARGO-YBJ experiment. On the other hand the larger $N_{h i t}$, the better the angular resolution. Assuming two different GRB Ecuts $(100 \mathrm{GeV}$ and $1000 \mathrm{GeV})$, the optimal $N_{\text {hit }}$ range and angular window

\footnotetext{
1 Speaker: S.Z Chen, chensz@ihep.ac.cn
} 

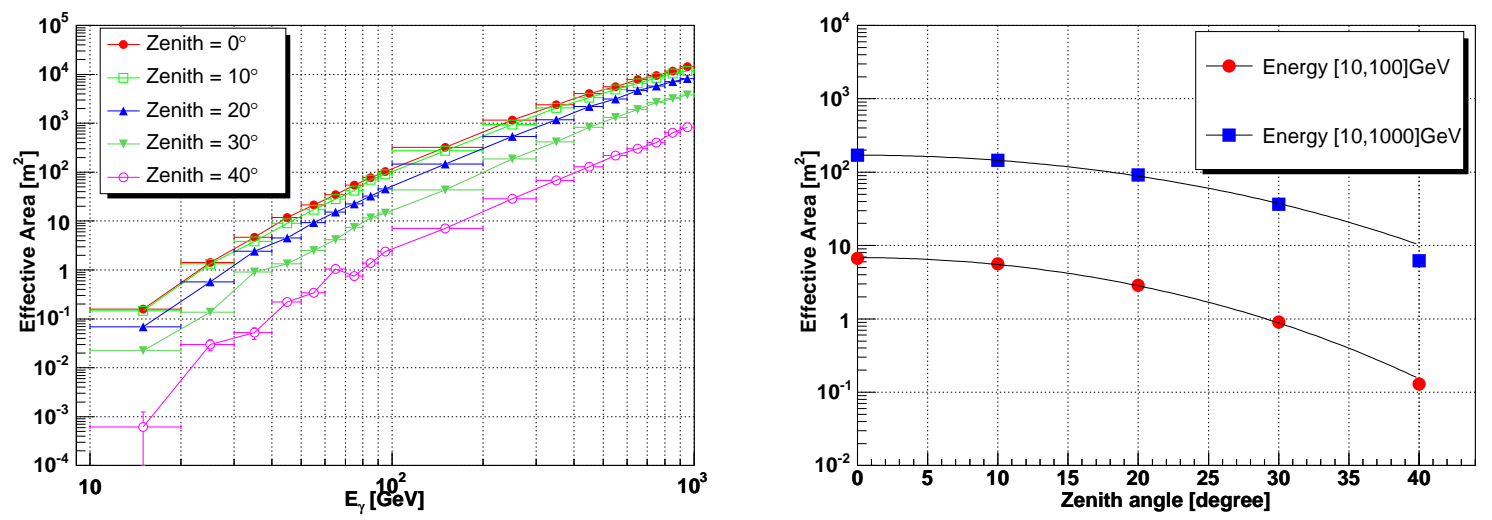

FIGURE 1. Left: Effective area of ARGO for gamma rays as a function of energy for five different zenith angles. Right: The average effective area in different energy range as a function of zenith angles. The lines are the fitting result using function $A_{0} \cos ^{n} \theta$.

TABLE 1. List of $N_{\text {hit }}$ range and the angular window size.

\begin{tabular}{rrr}
\hline \multicolumn{1}{c}{ Ecut } & $N_{\text {hit }}$ & $\phi_{70}$ \\
\hline $100 \mathrm{GeV}$ & $20-60$ & $3.8^{\circ}$ \\
$1000 \mathrm{GeV}$ & $20-500$ & $2.6^{\circ}$ \\
\hline
\end{tabular}

size to be used in the data analysis are further studied by MC simulation with the results shown in Table.1. These parameters are not sensitive to the GRB spectrum index.

During the data analysis, events are selected by the $N_{h i t}$ according to Table.1. An angular window surrounding the candidate GRB with an open angle in Table.1 is taken as the on-source one, in which the number of background events is estimated over two hours around the GRB trigger time using the "direct integral method" [11]. The left of Fig.2 shows the event rates of background detected in two different data selection conditions as a function of the incident zenith angle. Combining this result and MC simulation above, the $5 \sigma$ sensitivities to detect the GRBs with different durations $T_{\text {win }}$ and different incident zenith angles in two energy range [10,100] GeV and [10,1000] GeV are estimated and shown in the right of Fig.2. The sensitivity for GRBs with duration 20s at $\theta=20^{\circ}$ is $10^{-5} \mathrm{erg} / \mathrm{cm}^{2}$ in the energy range $[10,1000] \mathrm{GeV}$, and this is comparable with the fluence in $\mathrm{keV}$ band detected by satellite. The GRB counterpart is first searched in the T90 time, after that, an extended search is performed in different time intervals $1 \mathrm{~s}, 6 \mathrm{~s}, 12 \mathrm{~s}$, $24 \mathrm{~s}, 48 \mathrm{~s}$ and $96 \mathrm{~s}$ with running step $1 \mathrm{~s}, 2 \mathrm{~s}, 3 \mathrm{~s}, 6 \mathrm{~s}, 12 \mathrm{~s}$ and $24 \mathrm{~s}$ respectively over a period of two hours around the GRB trigger time.

\section{RESULT AND DISCUSSION}

The data used in this work was collected in the period from July 2006 to July 2008 , during which the DAQ was stopped from July 2007 to November 2007 for detector maintenance. 20 GRBs detected by satellites were within the FOV of ARGO-YBJ during the live time. Analysis result shows no significant excess for any of them. The fluence upper limits of VHE emission in the GRB T90 time window at 99\% C.L. are estimated by means of the Helene method [12] assuming a power-law spectrum index alpha=-2. In order to obtain a more realistic upper limit for the origin field of GRB, the optical depths predicted in [13](line P0.55) are used to remove the effect due to the absorption of the extragalactic background light (EBL). The upper limits for the $5 \mathrm{GRBs}$ with redshift information have been corrected, and the results are listed in Table 2. For the other 15 GRBs listed in Table 2 haven't redshift information, the correction for $[10,100] \mathrm{GeV}$ could be ignored, but correction factors for $[10,1000] \mathrm{GeV}$ are $1.36,1.75,5.10$ and 7.98 when redshifts are $0.05,0.1,0.5$ and 1.0 assuming the zenith angle $20^{\circ}$. For comparison, the fluence in the keV band measured by satellite is also given in Table 2. There is no data during some GRBs T90 time, but with data in the extend search phase, so they are listed here without upper limits. As an example, Fig.3 shows the comparison between the 

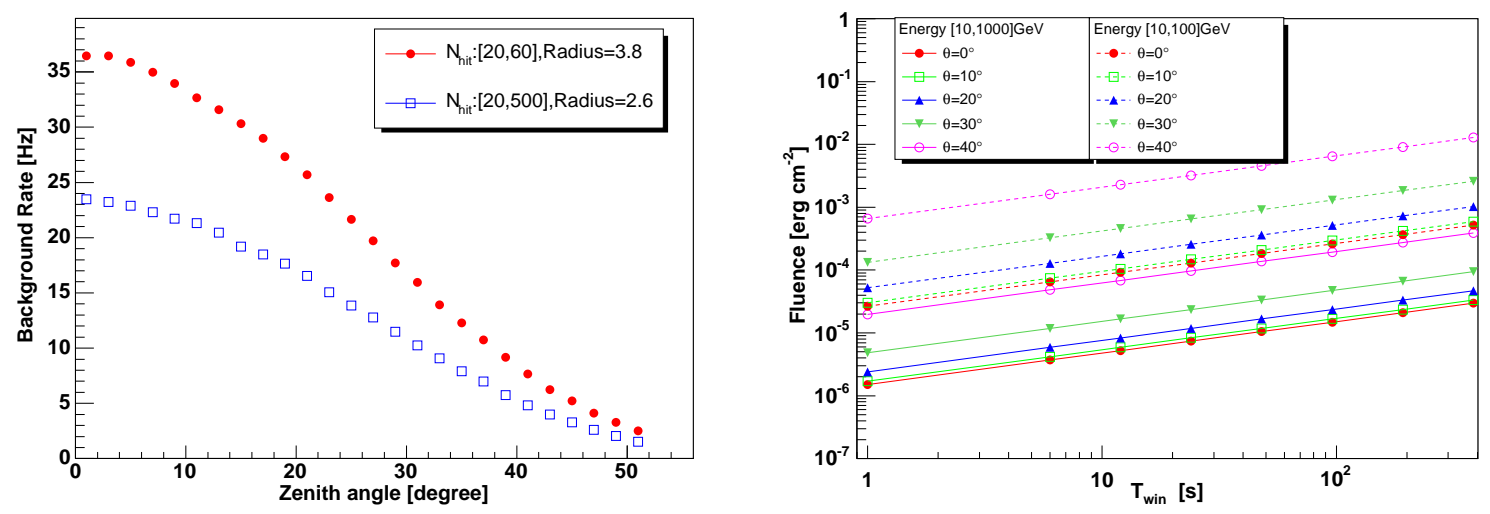

FIGURE 2. Left: The background rate detected in two different data selection conditions as a function of the incident zenith angle. Right:The $5 \sigma$ sensitivity as a function of GRB duration $T_{w i n}$ in two energy range $[10,100] \mathrm{GeV}$ and $[10,1000] \mathrm{GeV}$ for different incident zenith angles.

TABLE 2. List of GRBs in FOV $\left(\theta<45^{\circ}\right)$ of ARGO and 99\% C.L. fluence upper limit

\begin{tabular}{|c|c|c|c|c|c|c|c|}
\hline GRB & Instr. & redshift & $\begin{array}{r}\text { T90 } \\
s\end{array}$ & $\begin{array}{r}\theta \\
d e g\end{array}$ & $\begin{array}{r}\text { keV fluence } \\
\mathrm{erg} * \mathrm{~cm}^{-2}\end{array}$ & $\begin{array}{r}\mathbf{1 0 - 1 0 0 G e V} \\
\operatorname{erg} * \mathrm{~cm}^{-2}\end{array}$ & $\begin{array}{r}10-1000 G e V \\
e r g * \mathrm{~cm}^{-2}\end{array}$ \\
\hline 060714 & Swift & 2.71 & 115 & 42.8 & $2.9 \mathrm{E}-6(15-150)$ & $5.63 \mathrm{E}-3$ & $2.24 \mathrm{E}-3$ \\
\hline 060717 & Swift & $\ldots$ & 3 & 7.4 & $6.5 \mathrm{E}-8(15-150)$ & $\ldots$ & $\ldots$ \\
\hline 060801 & Swift & $\ldots$ & 0.5 & 16.8 & $8.0 \mathrm{E}-8(15-150)$ & $\ldots$ & \\
\hline 060805B & IPN & $\ldots$ & 8 & 29.1 & $1.1 \mathrm{E}-4(30-10000)$ & $1.29 \mathrm{E}-4$ & $5.08 \mathrm{E}-6$ \\
\hline 060807 & Swift & & 43.3 & 12.4 & $8.5 \mathrm{E}-7(15-150)$ & $7.32 \mathrm{E}-5$ & 4.23E-6 \\
\hline 060927 & Swift & 5.47 & 22.6 & 31.6 & $1.2 \mathrm{E}-6(15-150)$ & $6.21 \mathrm{E}-4$ & $3.04 \mathrm{E}-4$ \\
\hline 061028 & Swift & & 106 & 42.5 & 9.7E-7 (15-150) & $6.23 \mathrm{E}-3$ & $1.08 \mathrm{E}-4$ \\
\hline 061110A & Swift & 0.76 & 41 & 37.3 & $1.1 \mathrm{E}-6(15-150)$ & $1.18 \mathrm{E}-3$ & $1.74 \mathrm{E}-4$ \\
\hline 061122 & Integral & $\ldots$ & 18 & 33.5 & $2.3 \mathrm{E}-5(20-2000)$ & $4.27 \mathrm{E}-4$ & $8.45 \mathrm{E}-6$ \\
\hline 070201 & IPN & $\ldots$ & 0.15 & 20.6 & 2.0E-5 (20-2000) & $\ldots$ & .. \\
\hline 070219 & Swift & $\ldots$ & 17 & 39.3 & $3.2 \mathrm{E}-7(15-150)$ & & \\
\hline 070306 & Swift & 1.50 & 210 & 19.9 & $5.5 \mathrm{E}-6(15-150)$ & $3.53 \mathrm{E}-4$ & $1.37 \mathrm{E}-4$ \\
\hline 070531 & Swift & $\ldots$ & 44 & 44.3 & $1.1 \mathrm{E}-6(15-150)$ & $3.09 \mathrm{E}-3$ & $7.82 \mathrm{E}-5$ \\
\hline 070615 & Integral & $\ldots$ & 30 & 37.6 & & $1.42 \mathrm{E}-3$ & 3.93E-5 \\
\hline $071112 \mathrm{C}$ & Swift & 0.82 & 15 & 22.1 & 3.0E-6 (15-150) & $1.81 \mathrm{E}-4$ & $5.77 \mathrm{E}-5$ \\
\hline 080207 & Swift & $\ldots$ & 293 & 27.7 & $6.3 \mathrm{E}-6(15-150)$ & $6.78 \mathrm{E}-4$ & $2.84 \mathrm{E}-5$ \\
\hline 080328 & Swift & $\ldots$ & 90.6 & 37.2 & 9.4E-6 (15-150) & $1.60 \mathrm{E}-3$ & $4.79 \mathrm{E}-5$ \\
\hline 080515 & Swift & $\ldots$ & 21 & 43.2 & $2.0 \mathrm{E}-6(15-150)$ & $2.79 \mathrm{E}-3$ & $6.57 \mathrm{E}-5$ \\
\hline 080613B & Swift & $\ldots$ & 105 & 39.2 & $5.8 \mathrm{E}-6(15-150)$ & $2.49 \mathrm{E}-3$ & $7.32 \mathrm{E}-5$ \\
\hline 080727C & Swift & $\ldots$ & 79.7 & 34.5 & 5.3E-6(15-150) & $8.15 \mathrm{E}-4$ & $3.18 \mathrm{E}-5$ \\
\hline
\end{tabular}

upper limits and the simple extrapolation from the measurement in $\mathrm{keV}$ band, with the upper limit for GRB060805B is also calculated with assuming $\alpha=-2.5$.

It is known that the GRB spectra have a break at energy $E_{0}$, which distributes between $100 \mathrm{keV}$ and $1 \mathrm{MeV}$, and the average index $\alpha$ before the break is $\sim-1$ and the index $\beta$ beyond the break is $\sim-2.3$. But due to the energy band limitation, many of Swift GRBs measurements are without break information. So if a simple extrapolation with assuming $E_{0}=150 \mathrm{keV}$ and $\beta=-2$ is used, the upper limits for $[10,1000] \mathrm{GeV}$ derived by this work are lower (see Fig.3). If this extrapolation is more soft like $\beta=-2.3$, the upper limit would be higher, but this limit would be still useful in constraining GRB models when a double-peak shape extending into the VHE band is expected.

In conclusion, we have searched the counterparts, prompt, delayed and prior GeV-TeV emission, of 20 GRBs in the FOV of ARGO in about two years of effective operation. Although no significant excess is found, the upper limits are still useful to constrain GRB models in VHE emission. Up to now, our knowledge about the VHE emission from GRBs is still very limited, especially the existence of the VHE emission in GRB is still an open question. Thanks to 

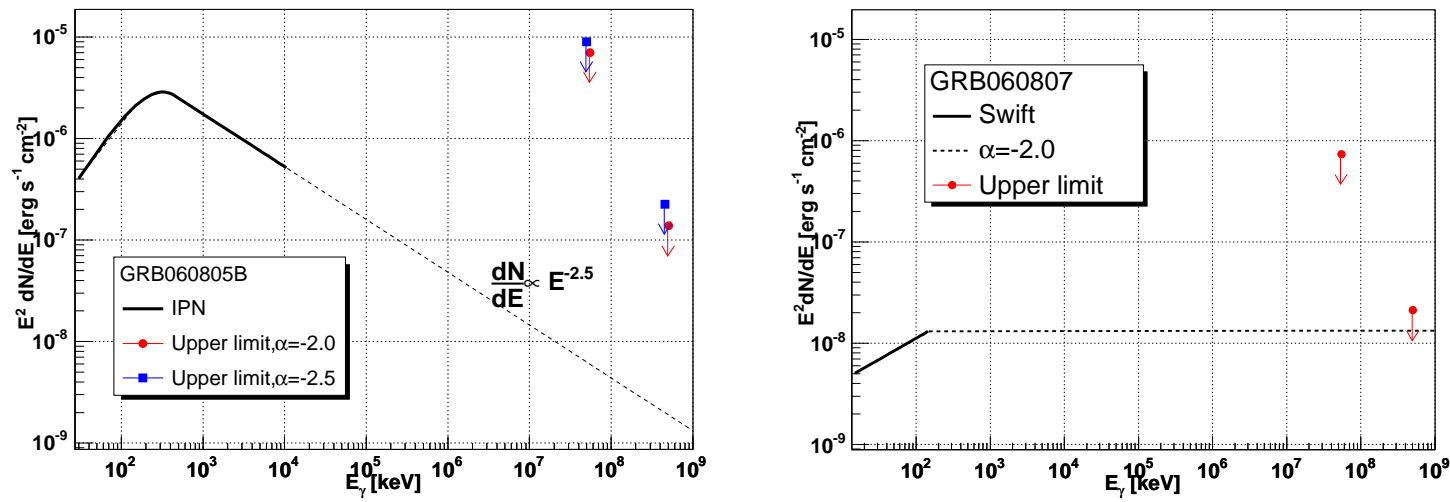

FIGURE 3. Upper limits got by ARGO and the simple extrapolations of GRB spectrums got by satellite. The upper limit for GRB060805B is also calculated assuming $\alpha=-2.5$.

the successful launch of GLAST, our understanding of GRB VHE emission would be greatly improved in the future.

\section{ACKNOWLEDGEMENTS}

This work is supported in China by NSFC(10120130794), the Chinese Ministry of Science and Technology, the Chinese Academy of Sciences, the Key Laboratory of Particle Astrophysics, CAS, and in Italy by the Istituto Nazionale di Fisica Nucleare (INFN).

\section{REFERENCES}

1. Hurley, et al. Nature 372, 652 (1994).

2. Gonzalez, et al. Nature 424, 749 (2003).

3. P. Meszaros, Rep. Prog. Phys. 69, 2259 (2006).

4. Amenomori, M. et al. A.A. 311, 919 (1996).

5. Atkins, R. et al. APJ 533, L119 (2000).

6. Padilla, L. et al.A.A. 337, 43 (1998).

7. Poirier,J.et al. Phys. Rev. D 67, 042001 (2003).

8. Albert, J. et al. APJ 641, L9 (2006).

9. Aielli, G. et al. Nucl. Instr. and Meth. A 562, 92 (2006).

10. D. Martello et al. ARGO Detector with GEANT3 package, 2000.

11. Fleysher, R. et al. APJ 603, 355 (2004).

12. Helene, O. et al. Nucl. Instr. Meth. 212, 319 (1983).

13. Aharonian, F. et al. Nature 440,1018 (2006). 